\title{
Quadratic Model for Predicting the Hardness of Heat Affected Zone in Water Cooled Cast Iron Weldment In Relation to Similarly Cooled Aluminum and Mild Steel Weldments
}

\author{
C. I. Nwoye \\ Department of Materials and Metallurgical Engineering, Federal University of Technology, \\ P.M.B 1526, Owerri, Nigeria. \\ Contact:chikeyn@yahoo.com
}

\begin{abstract}
Quadratic and linear models have been derived for predicting the heat-affected zone (HAZ) hardness of water cooled cast iron weldment in relation to the combined and respective values of the heat-affected zone hardness of aluminum and mild steel welded and cooled under the same conditions. It was found that the validity of the model is rooted on the fractional expression; $\gamma / 3.0749 \theta+\gamma / 3.0749 \beta+\theta / 3.0749 \beta=1$. The respective deviations of the model-predicted heataffected zone hardness values of aluminum, cast iron and mild steel from the corresponding experimental values were less than $0.01 \%$ which is quite insignificant, indicating reliability of the model.
\end{abstract}

Keywords: Model, Hardness, Heat Affected Zone, Cast Iron Weldments, Aluminum, Mild Steel.

\section{INTRODUCTION}

Research reports $[1,2]$ have shown that there are several processes and methods of arc welding including carbon-arc welding, atomic hydrogen welding, shielded metal arc welding, plasma arc welding, electroslag welding. It has been demonstrated that arc welding involves the process where by the heat generated by the electric arc is maintained in most cases between the electrodes and the work piece [3]. The quantity of heat required for melting the base metal in the vicinity of the arc and also the electrode is supplied by the arc. In arc welding, some of the processes utilize consumable electrodes which serve to strike an arc onto the work pieces, and they melt to provide the weld metal. In recent times, advancement has been made in such joining processes as adhesives, mechanical fasteners, brazing soldering [4]. However, welding remains the most important metal joining process.

It is generally believed that arc welding is the most widely used fusion welding process. It produces smooth welding surfaces and utilizes both direct and alternating current. Oxidation is minimal as weld metal is completely shielded from the atmosphere. The process is excellent welding low carbon, medium carbon and alloy steels. The arc is quiet, discomfort from glare or 
fume is minimal, and is applicable in fabricating vessels, boilers and pipes, etc. Disadvantages of the process include need for very high current for welding operations and formation of a crater in the molten metal of the work piece arising from the pressure produced by the stream of ions flowing from the cathode [2]. Electrodes are the elements of an arc lamp or furnace between which an arc is struck. They are filler materials which a joining engineer should be able to match with the parent material to avoid failure [1]. Uncoated electrodes produce an atmosphere of oxygen and nitrogen, so that the oxides and nitrides formed may be in the weld metal, thus impairing ductility and impact toughness in the weld. The situation is avoided by use of coated electrodes, which contains slag and so form a fluid covering over the weld [2]. In this case, stabilization of the arc is achieved by including materials which would produce ionization and consequently may be wielded by the metallic arc process. In welding carbon and low carbon steels, coated electrodes are used especially for low carbon steels but for alloy steels in which martensite occurrence is likely on cooling and formation of hydrogen embrittlement expected, the electrode coating must be free from hydrogen forming cellulose [5].

Cracking of weldment has been found [6] to be one of the reasons for low mechanical properties such as hardness and impact strength in welded parts. Adjacent to the immediate welded area or fusion zone is the heat affected zone [6]. The mechanical property of main importance in HAZ is the hardness since it gives an indication of the degree of embrittlement there. Studies [7] have shown that the heat affected zone hardness produced by any given welding operation depends on the cooling rate experienced by the HAZ. Too rapid rate of cooling favours the formation of hard and brittle martensite in all the sub zones of the HAZ or increases the martensite region in size relative to the other regions. The presence of martensite in the HAZ results in a very high hardness value for the heat affected zone. Slow cooling favours a better microstructure needed for engineering applications. Also, the more rapid the quenching rate, the greater the HAZ hardness.

Several literatures have reported studies carried out on different joining processes and methods, but no emphasis has been placed on the derivation of models for prediction or evaluation of the hardness of the heat affected zone (HAZ) in weldments cooled in different media; evaluation of the hardness of HAZ cooled in a particular medium as a function of the hardness of HAZ from the same material but cooled in different media. Researches carried out on HAZ; its cooling and mechanical properties have not addressed the issue of predicting or evaluating the hardness of the HAZ of a material cooled in a particular medium by simple substitution of the value of the hardness of HAZ from the same material, but cooled in different media. The hardness of HAZ in aluminum, cast iron and mild steel cooled in kerosine was found to be exactly the same as the hardness value of the same materials cooled in groundnut oil [8]. This implies that

$$
\mathrm{H}_{\mathrm{G}}=\mathrm{H}_{\mathrm{K}}
$$

Where

$$
\begin{aligned}
& \mathrm{H}_{\mathrm{G}}=\text { Hardness of HAZ cooled in groundnut oil } \\
& \mathrm{H}_{\mathrm{K}}=\text { Hardness of HAZ cooled kerosine }
\end{aligned}
$$

It has been reported [8] that $8-10 \%$ less hardness than that from water occurs when kerosine or groundnut oil is used as quenchant for HAZ. He discovered that quenching the HAZ with 
kerosine or groundnut oil gives approximately $8-10.7 \%$ more hardness than that from quenching with air. He found that palm oil gave the lowest hardness and cooling rate on the HAZ.

The present study aims at deriving quadratic and linear models for predicting the hardness of the heat affected zone (HAZ) in cast iron weldment cooled in water, as a function of the respective and combined values of HAZ hardness of aluminum and mild steel welded and cooled under the same conditions.

\section{MATERIALS AND METHODS}

Aluminum, mild steel and cast iron were cut and welded using the shielded metal arc welding technique and the hardness of the HAZ (cooled in water maintained at room temperature) tested. The hardness of the HAZ is as presented in Table 2. The full details of the experimental procedures and equipment used are presented in the previous report [8]. Table 1 shows the welding current and voltage used.

Table 1. Variation of materials with welding current and voltage [8]

\begin{tabular}{|l|l|c|c|}
\hline Materials & Current Type & \multicolumn{1}{|l|}{$\begin{array}{l}\text { Welding } \\
\text { Current }\end{array}$} & $\begin{array}{l}\text { Welding } \\
\text { (V) }\end{array}$ \\
\hline Aluminum & Direct (d.c) & 120 & 280 \\
Cast Iron & Alternating & 180 & 220 \\
Mild Steel & (a.c) & 180 & 220 \\
& Alternating & & \\
& (a.c) & & \\
\hline
\end{tabular}

Table 2. Hardness of HAZ in weldments [8]

\begin{tabular}{|l|l|}
\hline Materials & $\begin{array}{l}\text { HAZ } \\
(\mathrm{VHN})\end{array}$ \\
\hline Aluminum & 458 \\
Cast Iron & 1010 \\
Mild Steel & 560 \\
& \\
\hline
\end{tabular}

\section{MODEL FORMULATION}

Experimental data obtained from research work [8] carried out at Metallurgical and Materials Engineering Department of Federal University of Technology, Owerri were used for this work. Results of the experiment as presented in the report [8] and used for the model formulation are as shown in Table 2. Computational analysis of the experimental data [8] shown in Table 2 resulted in Table 3. 
Table 3. HAZ Hardness ratio between aluminum, mild steel, and cast iron weldments cooled in water.

\begin{tabular}{|c|l|l|}
\hline $\begin{array}{l}\text { Ratio of } \\
\text { symbols } \\
\text { designating } \\
\text { HAZ hardness }\end{array}$ & $\begin{array}{l}\text { Ratio of HAZ } \\
\text { hardness } \\
\text { values }\end{array}$ & $\begin{array}{l}\text { Results of the } \\
\text { Ratio of HAZ } \\
\text { hardness values }\end{array}$ \\
\hline$\gamma / \theta$ & $458 / 1010$ & 0.4535 \\
$\theta / \beta$ & $1010 / 560$ & 1.8035 \\
$\gamma / \beta$ & $458 / 560$ & 0.8179 \\
\hline
\end{tabular}

Table 3 shows that the hardness of HAZ in cast iron weldment cooled in water is a function of the hardness of HAZ in aluminum and mild steel weldment also cooled in water, hence

$$
\text { Therefore } \begin{aligned}
\gamma & =0.4535 \theta \\
\theta & =2.2051 \gamma \\
\theta & =1.8035 \beta \\
\gamma & =0.8179 \beta
\end{aligned}
$$

Also form Table 3,

$$
\begin{gathered}
\left(\frac{\gamma}{\theta}\right)+\left(\frac{\theta}{\beta}\right)+\left(\frac{\gamma}{\beta}\right)=0.4535+1.8035+0.8179 \\
\left(\frac{\gamma \beta+\gamma \theta+\theta^{2}}{\theta \beta}\right)=3.0749 \\
\gamma \beta+\gamma \theta+\theta^{2}=3.0749 \theta \beta
\end{gathered}
$$

Dividing both sides of equation (8) by $3.0749 \theta \beta$

$$
\left(\frac{\gamma}{3.0749 \theta}\right)+\left(\frac{\gamma}{3.0749 \beta}\right)+\left(\frac{\theta}{3.0749 \beta}\right)=1
$$

Also from equation (8)

$$
\begin{aligned}
& \theta^{2}+\gamma \theta-3.0749 \theta \beta+\gamma \beta=0 \\
& \theta^{2}+(\gamma-3.0749 \beta) \theta+\gamma \beta=0
\end{aligned}
$$

Solving the quadratic equation in equation (8) for the value of $\theta$

$$
\theta^{2}+(\gamma-3.0749 \beta) \theta=-\gamma \beta
$$

Adding square of the half of the coefficient of $\theta$ to both sides of equation (12)

$$
\begin{gathered}
\theta^{2}+(\gamma-3.0749 \beta) \theta+\left(\frac{\gamma-3.0749 \beta}{2}\right)^{2}=-\gamma \beta+\left(\frac{\gamma-3.0749 \beta}{2}\right)^{2} \\
\left(\frac{\theta+\gamma-3.0749 \beta}{2}\right)^{2}=-\gamma \beta+\left(\frac{\gamma-3.0749 \beta}{2}\right)^{2}
\end{gathered}
$$




$$
\begin{aligned}
& \left(\theta+\frac{\gamma-3.0749 \beta}{2}\right)=\sqrt{ }\left(\left(\frac{\gamma-3.0749 \beta}{2}\right)^{2}-\gamma \beta\right) \\
& \theta=-\left(\frac{\gamma-3.0749 \beta}{2}\right)+\sqrt{ }\left(\left(\frac{\gamma-3.0749 \beta}{2}\right)^{2}-\gamma \beta\right) \\
& \theta=\left(\frac{3.0749 \beta-\gamma}{2}\right)+\sqrt{ }\left(\left(\frac{\gamma-3.0749 \beta}{2}\right)^{2}-\gamma \beta\right)
\end{aligned}
$$

The derived model is equation (17)

Where $\gamma=$ Model-predicted hardness of HAZ in aluminum weldment cooled in water (VPN)

$\beta=$ Model-predicted hardness of HAZ in mild steel weldment cooled in water (VPN) $\theta=$ Model-predicted hardness of HAZ in cast iron weldment cooled in water (VPN)

\section{BOUNDARY AND INITIAL CONDITIONS}

The welding was carried out under atmospheric condition. After welding, weldments were also maintained under atmospheric condition. Welding current and voltage used are $180 \mathrm{~A}$ and $220 \mathrm{~V}$ respectively. $\mathrm{SiO}_{2}$-coated electrodes were used to avoid oxidation of weld spots. The coolants used were maintained at $25^{\circ} \mathrm{C}$ (room temperature). Volume of coolants used; $1000 \mathrm{~cm}^{3}$. No pressure was applied to the HAZ during or after the welding process. No force due to compression or tension was applied in any way to the HAZ during or after the welding process. The sides and shapes of the samples are symmetries.

\section{MODEL VALIDATION}

The derived model was validated by evaluating the model-predicted values of HAZ hardness in cast iron weldment $\theta$ cooled in water and comparing them with the corresponding values obtained from the experiment $\theta_{\exp }$ [8]. Following re-arrangement of the model equation; (17), the values of $\gamma$ and $\beta$ were also evaluated as;

$$
\begin{aligned}
& \gamma=\left(\frac{3.0749 \theta \beta-\theta^{2}}{\beta+\theta}\right) \\
& \beta=\left(\frac{\gamma \theta+\theta^{2}}{3.0749 \theta-\gamma}\right)
\end{aligned}
$$

and compared with their respective corresponding experimental values $\gamma_{\exp }$ and $\beta_{\exp }$ to further establish the validity of the model. The model-predicted values of $\theta, \gamma$ and $\beta$ are shown in Table 5. The general model was also validated by solving the derived quadratic expression (equation (11)) for the value of $\theta$ using the conventional general formular; $x=\left[-b \pm \sqrt{ }\left(b^{2}-4 a c\right)\right] / 2 a[9]$ 
derived from the quadratic equation; $\mathrm{ax}^{2}+\mathrm{bx}+\mathrm{c}=0$. Therefore, for equation (11); $\theta^{2}+(\gamma-$ $3.0749 \beta) \theta+\gamma \beta=0, \quad a=1, b=\gamma-3.0749 \beta, c=\gamma \beta$ and $x=\theta$.

Analysis and comparison between the model-predicted values $\theta, \gamma, \beta$ and the respective corresponding experimental values $\theta_{\exp }, \gamma_{\exp }$, and $\beta_{\exp }$ reveal deviations of model data from the experimental data. This is attributed to the non-consideration of the chemical properties of the coolant and the physiochemical interactions between the materials (aluminum, mild steel and cast iron) and the coolant which is believed to have played vital roles in modifying the microstructure of the HAZ during the coolant process. These deviations necessitated the introduction of correction factor to bring the model-predicted values to exactly that of the corresponding experimental values.

Deviation (Dv) of the model-predicted HAZ hardness values $(\theta, \gamma$, and $\beta)$ from the corresponding experimental values $\theta_{\exp }, \gamma_{\text {exp }}$, and $\beta_{\exp }$ is given by

Where

$$
D v=\left(\frac{M_{H}-E_{H}}{E_{H}}\right) \times 100
$$

$\mathrm{M}_{\mathrm{H}}=$ Model-predicted HAZ hardness values

$\mathrm{E}_{\mathrm{H}}=\mathrm{HAZ}$ hardness values from the experiment [8]

Correction factor $(\mathrm{Cf})$ is the negative of the deviation i.e.

$$
\mathrm{Cf}=-\mathrm{Dv}
$$

Therefore

$$
C f=-100\left(\frac{M_{H}-E_{H}}{E_{H}}\right)
$$

Introduction of the values of $\mathrm{Cf}$ from equation (22) into the models give exactly the corresponding experimental values $\theta_{\text {exp }}, \gamma_{\text {exp }}$, and $\beta_{\exp }[8]$.

\section{RESULTS AND DISCUSSION}

A comparison of the HAZ hardness values from experiment and those of the model show model values very much within the range of the experimental values. Results of this comparison are presented in Tables 4 and 5. Model values of $\theta$ evaluated from equations (3) and (4) and tabulated in Table 4 show that the associated equations are valid since all of them gave almost the same corresponding experimental values $\theta_{\exp }$. The value of $\gamma$ in equation (5) was evaluated to establish the validity of the model. It was found that the model-predicted $\gamma$ value was also almost

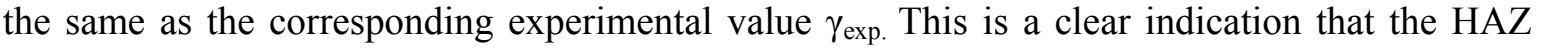
hardness of any of aluminum, mild steel and cast iron weldments cooled in water can be predicted as a function of the HAZ hardness of any of the other two materials, providing each pair was cooled in water. Table 5 also indicates that the model-predicted value of $\beta$ is approximately the same as the corresponding experimental value. 
Table 4. Comparison of the hardness of HAZ in aluminum, mild steel and cast iron weldments cooled in water as obtained from experiment [8] and as predicted by derived model (each material as a function of 1-material).

\begin{tabular}{|c|c|c|c|c|c|}
\hline $\mathrm{N}$ & Models derived & $\mathrm{M}_{\mathrm{H}}$ & $\mathrm{E}_{\mathrm{H}}$ & $\mathrm{Dv}(\%)$ & $\mathrm{Cf}(\%)$ \\
\hline 1 & $\theta=2.2051 \gamma$ & 1009.94 & 1010 & -0.0059 & +0.0059 \\
1 & $\gamma=0.8179 \beta$ & 458.02 & 458 & +0.0044 & -0.0044 \\
1 & $\theta=1.8035 \beta$ & 1009.96 & 1010 & -0.0040 & +0.0040 \\
\hline
\end{tabular}

Where

$\mathrm{N}=$ No. of materials constituting the corresponding model as independent variable.

It can also be seen from Table 5 that the model-predicted values of $\gamma$ and $\beta$ are also almost the same as the corresponding experimental values of $\gamma_{\exp }$ and $\beta_{\exp }$ respectively. The value of $\theta$ (1010.0045 VPN) evaluated using the general formular for quadratic equation was exactly equal to that predicted by the general model (equation (17)). Tables 4 and 5 indicate that the respective deviations of the model-predicted HAZ hardness values $\theta, \gamma$ and $\beta$ from those of the corresponding experimental values $\theta_{\exp }, \gamma_{\exp }$, and $\beta_{\exp }$ are all less than $0.01 \%$ which is quite negligible and within the acceptable model deviation range from experimental results.

Table 5. Comparison of the hardness of HAZ in aluminum, mild steel and cast iron weldments cooled in water as obtained from experiment [8] and as predicted by derived model (each material as a function of 2-materials).

\begin{tabular}{|c|c|c|c|c|c|}
\hline $\mathrm{N}$ & Models derived & $\mathrm{M}_{\mathrm{H}}$ & $\mathrm{E}_{\mathrm{H}}$ & $\mathrm{Dv}(\%)$ & $\mathrm{Cf}(\%)$ \\
\hline 2 & $\theta=[3.0749 \beta-\gamma] / 2+\sqrt{[((\gamma-}$ & 1010.0045 & 1010 & +0.0004 & -0.0004 \\
& $\left.3.0749 \beta) / 2)^{2}-\gamma \beta\right]$ & & & & \\
2 & $\gamma=\left[3.0749 \theta \beta-\theta^{2}\right] / \beta+\theta$ & 458.0022 & 458 & +0.0005 & -0.0005 \\
2 & $\beta=\left[\gamma \theta+\theta^{2} / 3.0749 \theta-\gamma\right]$ & 559.9987 & 560 & -0.0002 & +0.0002 \\
\hline
\end{tabular}

Furthermore, the values of $\gamma$ and $\beta$ (from equations (18) and (19) respectively) evaluated to be approximately equal to the respective corresponding experimental values $\gamma_{\exp }$ and $\beta_{\exp }$ confirm the validity of the model. This also implies that the general model; equation (17) can predict the HAZ hardness of any of aluminum, mild steel and cast iron weldments cooled in water as a function of the HAZ hardness of the other two materials, providing the three materials (aluminum, mild steel and cast iron) constituting the model were cooled in water. Equation (17) is regarded as the general model equation because it comprised the HAZ hardness of all the materials considered for the model formulation. It was found that the validity of the model is rooted on the fractional expression; $\gamma / 3.0749 \theta+\gamma / 3.0749 \beta+\theta / 3.0749 \beta=1$ since the actual computational analysis of the expression was also equal to 1 apart from the fact that the 
expression comprised the three metallic materials. Based on the foregoing, the models in equations (3), (4) and (17) are valid and very useful for predicting HAZ hardness of aluminum, mild steel and cast iron weldments cooled in water depending on the material of interest and the given HAZ hardness values for the other materials. The general model (equation (17)) was also found to give lesser magnitude of deviation from experimental HAZ hardness values and is therefore preferred to other derived models (equations (3) and (4)). However, the latter models will be much useful if the HAZ hardness is expected to be predicted in relation to just one material which may be either aluminum, mild steel or cast iron.

\section{CONCLUSION}

The derived models; $\theta=2.2051 \gamma$ and $\theta=1.8035 \beta$ can predict the HAZ hardness of cast iron weldment cooled in water as a function of the HAZ hardness of aluminum or mild steel welded and cooled under the same conditions. Similarly, the general model; $\theta=[3.0749 \beta-\gamma] / 2+\sqrt{ }[((\gamma-$ $3.0749 \beta) / 2)^{2}-\gamma \beta$ ] can predict the HAZ hardness of cast iron weldment cooled in water as a function of the HAZ hardness of both aluminum and mild steel welded and cooled under the same conditions. Furthermore, re-arrangement of these models could be done to evaluate the HAZ hardness of aluminum and mild steel respectively as in the case of cast iron. The validity of the model was rooted on the fractional expression; $\gamma / 3.0749 \theta+\gamma / 3.0749 \beta+\theta / 3.0749 \beta=1$ since the actual computational analysis of the expression was also equal to 1 . The respective deviations of the model-predicted HAZ hardness values $\theta, \gamma$, and $\beta$ from the corresponding experimental values $\theta_{\exp } \gamma_{\exp }$ and $\beta_{\exp }$ was less $0.01 \%$ indicating the reliability and validity of the model.

\section{ACKNOWLEDGEMENT}

The author thanks the management of Federal University of Technology, Owerri for providing the equipment used for this work.

\section{REFERENCES}

[1] Davies, A. C., (1993). The Science and Practice of Welding, $10^{\text {th }}$ Edition, Cambridge University

Press, p521.

[2] Higgins, R. A., (1994). Engineering Metallurgy, Arc Welding Processes, $2^{\text {nd }}$ Edition, Edward Arnold Publishers, London.p57.

[3] Lancaster, J. F., (1993). Metallurgy of Welding $5^{\text {th }}$ Edition, London. Chapman and Hall p389.

[4] Callister, W.D., (Jnr.) (1996). Material Science and Engineering. An introduction, $1^{\text {st }}$ Edition, Sydney John Wiley \& Sons inc. p852.

[5] Higgins, R. A., (1977). Engineering Metallurgy Part II, $2^{\text {nd }}$ Edition, London, ELBS, p466.

[6] Nnuka, E. E., Ovat, F. A., and Oseni, M. I., (2008). Effect of Electrode Types on the Mechanical Properties and Structure of Welded Steel Joints. JMME Vol. 3, No. 1.

[7] Lancaster, J. F., (1987). Metallurgy of Welding: The Mechanical Properties of the Heat Affected Zone, $4^{\text {th }}$ Edition, Allen and University, London. $\mathrm{p} 45$. 
[8] Nwoye, C. I. (2008). Comparative Studies of the Cooling Ability of Hydrocarbon Based Media and their Effects on the Hardness of the Heat Affected Zone (HAZ) in Weldments, JMME, Vol.3, No.1

[9] Stroud, K. A., (1986). Further Engineering Mathematics. $1^{\text {st }}$ edition, Macmillan Education, London. p47. 